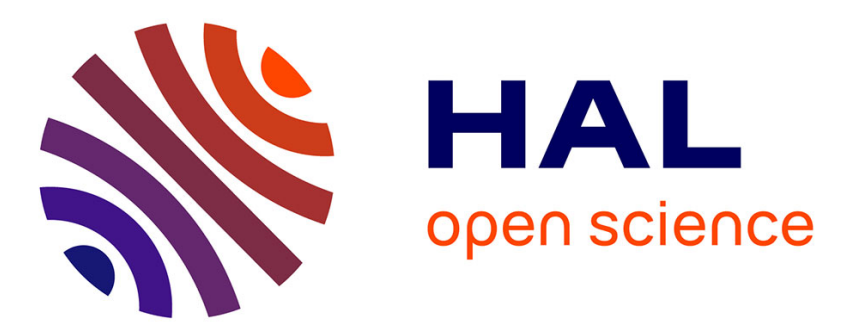

\title{
Meissner to vortex phase transition in a two-leg ladder in artificial gauge field
}

\author{
M Di Dio, R Citro, S de Palo, E Orignac, M.-L Chiofalo
}

\section{To cite this version:}

M Di Dio, R Citro, S de Palo, E Orignac, M.-L Chiofalo. Meissner to vortex phase transition in a two-leg ladder in artificial gauge field. The European Physical Journal. Special Topics, 2015, Novel Quantum Phases and Mesoscopic Physics in Quantum Gases, 224 (3), pp.525. 10.1140/epjst/e201502382-2 . ensl-01145026

\section{HAL Id: ensl-01145026 \\ https://hal-ens-lyon.archives-ouvertes.fr/ensl-01145026}

Submitted on 23 Apr 2015

HAL is a multi-disciplinary open access archive for the deposit and dissemination of scientific research documents, whether they are published or not. The documents may come from teaching and research institutions in France or abroad, or from public or private research centers.
L'archive ouverte pluridisciplinaire HAL, est destinée au dépôt et à la diffusion de documents scientifiques de niveau recherche, publiés ou non, émanant des établissements d'enseignement et de recherche français ou étrangers, des laboratoires publics ou privés.

$$
\text { Copyright }
$$


EPJ manuscript No.

(will be inserted by the editor)

\title{
Meissner to vortex phase transition in a two-leg ladder in artificial gauge field
}

\author{
M. Di Dio ${ }^{1}$, R. Citro $^{2}$, S. De Palo ${ }^{1,3}$, E. Orignac ${ }^{4}$, and M.-L. Chiofalo ${ }^{5}$ \\ 1 CNR-IOM-Democritos National Simulation Centre, UDS Via Bonomea 256, 34136 Trieste, Italy \\ 2 Dipartimento di Fisica "E. R. Caianiello", Università degli Studi di Salerno, Via Giovanni Paolo II, \\ Fisciano, Italy \\ 3 Dipartimento di Fisica, Università di Trieste, strada Costiera 11, 34151 Trieste, Italy \\ 4 Laboratoire de Physique de l'ENS-Lyon, CNRS UMR5672, 46 Allée d'Italie, 69007 Lyon, France \\ ${ }^{5}$ Dipartimento di Fisica and INFN, Università di Pisa, Largo Bruno Pontecorvo 3, 56127 Pisa, Italy
}

\begin{abstract}
We consider a two-leg boson ladder in artificial gauge field with hardcore intraleg and negligible interleg interactions. Using numerical simulations based on the Density Matrix Renormalization Group (DMRG) algorithm, combined with a bosonization approach, we study its commensurate-incommensurate transition to a vortex phase at a critical flux. We discuss the finite-size scaling behavior of the longitudinal current near the transition. For weak interchain boson hopping, the finite size scaling is in agreement with the predictions from bosonization.
\end{abstract}

\section{Introduction}

Ultracold atomic gases allow the simulation of condensed-matter physics model Hamiltonians such as the Bose Hubbard model [1] with a high tunability of the parameters. Recently, it has become possible to simulate the orbital effect of a magnetic field or a spin-orbit coupling using Raman processes [2-5]. In condensed matter physics, the two-leg bosonic ladder has been studied in relation with Josephson ladders [6-10]. For low magnetic field, the two-leg bosonic ladder is expected to show an analog of the Meissner state, in which currents circulate only along the legs of the ladder, while for higher field a vortex state is expected $[6,9]$. The phase transition between these two states is a commensurate-incommensurate transition [11,12]. However, actual Josephson junction ladders are dissipative systems $[13,14]$ and the resulting decoherence is expected to spoil the quantum phase transitions. Ultracold atomic gases have instead permitted the experimental realization of a two-leg bosonic ladder [15] under magnetic flux, allowing the observation of both the Meissner and the vortex phase in the case of non-interacting bosons. This has spurred recent theoretical activity [16-28] on the two-leg bosonic ladder in a magnetic field. In the present manuscript, we describe the commensurate-incommensurate transition from the Meissner to the vortex phase by a DMRG study combined with a bosonization approach and focus on the scaling behavior of the longitudinal current near the transition when a hard-core condition is established for the interaction along the legs. 


\section{Hamiltonian and bosonization approach}

The Hamiltonian of the symmetric two-leg boson Hubbard ladder subjected to a flux $\lambda$ per plaquette is [9]:

$$
\begin{aligned}
& H=-t \sum_{j, \sigma}\left[b_{j, \sigma}^{\dagger} e^{i \frac{\lambda}{2} \sigma} b_{j+1, \sigma}+b_{j+1, \sigma}^{\dagger} e^{-i \frac{\lambda}{2} \sigma} b_{j, \sigma}\right] \\
& +\Omega \sum_{j, \sigma} b_{j, \sigma}^{\dagger} b_{j,-\sigma}+\sum_{j, \sigma} U n_{j \sigma}\left(n_{j \sigma}-1\right)
\end{aligned}
$$

where $b_{j, \sigma}^{\dagger}\left(b_{j, \sigma}\right)$ is the boson creation (annihilation) at site $j$ on the leg labelled with $\sigma= \pm 1$, $t$ is the hopping amplitude along the legs, $\Omega$ the hopping amplitude between the rungs, and $U$ the on-site interaction. The density of bosons is $n_{j, \sigma}=b_{j, \sigma}^{\dagger} b_{j, \sigma}$. This Hamiltonian can also describe a two-component bosonic system ( $\sigma$ being then the spin quantum number) with a spin-orbit interaction. For the sake of clarity, we will always use the two-leg ladder description in the rest of the manuscript.

The low-energy physics of this system can be properly investigated using Haldane's bosonization of interacting bosons $[29,30]$ and treating $\Omega$ as a perturbation [9]. For generic incommensurate filling the low energy Hamiltonian can be written as $H=H_{c}+H_{s}^{\lambda}$ with

$$
\begin{aligned}
& H_{c}=\int \frac{d x}{2 \pi}\left[u_{c} K_{c}\left(\pi \Pi_{c}\right)^{2}+\frac{u_{c}}{K_{c}}\left(\partial_{x} \phi_{c}\right)^{2}\right]+2 g_{3} \cos \left(\sqrt{8} \phi_{c}+\delta x\right) \\
& H_{s}^{\lambda}=\int \frac{d x}{2 \pi}\left[u_{s} K_{s}\left(\pi \Pi_{s}\right)^{2}+\frac{u_{s}}{K_{s}}\left(\partial_{x} \phi_{s}\right)^{2}\right]-\Omega A_{0}^{2} \int d x \cos \sqrt{2} \theta_{s}-\frac{\lambda u_{s} K_{s}}{\sqrt{2} a} \int d x \Pi_{s}(x),
\end{aligned}
$$

where we introduced the symmetric (antisymmetric) $\phi_{c(s)}=\left(\phi_{1} \pm \phi_{-1}\right) / \sqrt{2}$ combination of the bosonized operator, together with its conjugate variables $\pi \Pi_{c(s)}=\left(\nabla_{x} \theta_{1}(x) \pm \nabla_{x} \theta_{-1}(x)\right) / \sqrt{2} . a$ is the lattice spacing along the legs of the ladder, $\delta=2 \pi(n-1) / a$, with $n$ the mean density and $A_{0}^{2}$ is some non-universal amplitude coming from the expansion of the density operator [29]. The Hamiltonian $H_{c}$, with linear spectrum, describes the leg-symmetric density excitations while $H_{s}$ describes the leg-antisymmetric density excitations.

In the case of hard-core bosons, for $\lambda=0$ [31] and at half-filling, a Mott gap opens up as soon as the hopping amplitude between the rungs is finite and the transition is of a KosterlitzThouless type. For other fillings the ground state is a gapless Luttinger liquid with a Luttinger parameter that depends on the filling [9]. $H_{s}^{\lambda}$, for $\lambda=0$, is the integrable quantum sine-Gordon model with a gapful spectrum for $K_{s}>1 / 4$. For $1 / 4<K_{s}<1 / 2$ the gapped excitations above the ground state are only solitons while for $K_{s}>1 / 2$ the gapped excitations include also soliton bound states called breathers [32].

For $\lambda \neq 0$, the ground state of the Hamiltonian (3) shows a commensurate-incommensurate (C-IC) quantum transition $[11,12]$ driven by the flux $\lambda$. This transition can be followed by examining the behavior of the longitudinal current in the antisymmetric sector which is linked by the Hellmann-Feynman theorem to the derivative of the ground state energy with respect to $\lambda$ :

$$
J_{s}=-i t \sum_{j, \sigma} \sigma\left(e^{i \lambda \sigma} b_{j, \sigma}^{\dagger} b_{j+1, \sigma}-e^{-i \lambda \sigma} b_{j+1, \sigma}^{\dagger} b_{j, \sigma}\right)
$$

and of the current perpendicular to the ladder

$$
J_{r, j}=i\left(b_{j, \sigma}^{\dagger} b_{j,-\sigma}-b_{j,-\sigma}^{\dagger} b_{j, \sigma}\right)
$$

For $\lambda$ under the threshold $\lambda_{c} \sim\left(\Omega A_{0}^{2} a / u_{s}\right)^{\frac{1}{2-1 /\left(2 K_{s}\right)}}$, the excitations above the ground state remain gapful. In such regime, $J_{s}=u_{s} K_{s} \lambda /(2 \pi)$ grows linearly with the imposed flux and $J_{r}=0$. This phase can be pictorially described as a Meissner phase where the current exists along the legs of the ladder and they screen out the applied magnetic field. When $\lambda$ exceeds the 


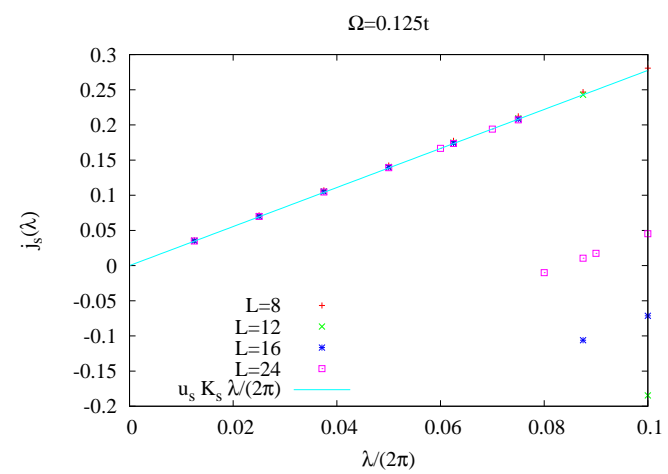

Fig. 1. A plot of current versus flux in the Meissner phase for system sizes $L=8,12,16,24$ and $\Omega=0.125 t$. The current responds linearly to the flux with $u_{s} K_{s} /(2 \pi)=2.77 \pm 0.02$ below the threshold $\lambda_{c}=0.408$. Above this value some extra contribution to the edge current developes and the linear behavior is lost.

threshold $\lambda_{c}$, it becomes energetically advantageous to populate the ground state of $H_{s}$ with a finite density of solitons to form a Tomonaga-Luttinger liquid. The low energy properties of this state are then described by the effective Hamiltonian:

$$
H_{s}^{*}=\int \frac{d x}{2 \pi}\left[u_{s}^{*}(\lambda) K_{s}^{*}(\lambda)\left(\pi \Pi_{s}^{*}\right)^{2}+\frac{u_{s}^{*}(\lambda)}{K_{s}^{*}(\lambda)}\left(\partial_{x} \phi\right)^{2}\right]
$$

In this phase both the currents along the legs and on the rungs are non-zero and a vortex phase similar to the one in a superconductor is formed. Near the transition point $\lambda_{c}$, it was found by fermionization [9] that $J_{s}=u_{s} K_{s}\left(\lambda-\sqrt{\lambda^{2}-\lambda_{c}^{2}}\right) /(2 \pi)$ and thus the current decreases on increasing the flux $\lambda$. Notice that the Hamiltonian (1) is invariant under the simultaneous transformations $\lambda \rightarrow \lambda+2 \pi$ and $b_{j, \sigma} \rightarrow(-1)^{j} b_{j \sigma}$. Under parity $b_{j, \sigma} \rightarrow b_{-j, \sigma}, H^{\lambda} \rightarrow H^{-\lambda}$. These symmetries imply that $J_{s}(\lambda+2 \pi)=J_{s}(\lambda)$ and $J_{s}(-\lambda)=-J_{s}(\lambda)$, so that it is enough to consider only $0<\lambda<\pi$. If $\lambda=\pi$ the longitudinal current $J_{s}$ is zero. An exact behavior of the current can be obtained by a DMRG treatment of the Hamiltonian (1), but in order to compare bosonization results with DMRG simulations a finite-size treatment is necessary, as illustrated below.

\section{Density Matrix Renormalization group and finite size scaling}

We performed simulations based on the DMRG algorithm [33,34] to investigate the hard core boson limit $(U \rightarrow+\infty)$ of (1) with a fixed density of $n=0.5$ and estimate the currents (4) and (5) using Periodic Boundary Conditions (PBC) for sizes $L=8,12,16,24$ and 48. Simulations are done keeping up to $m=1256$ states during the renormalization procedure in a way that the truncation error i.e. the weight of the discarded states, is at most of order $10^{-6}$, while the maximum error on the ground-state energy is of order $5 \times 10^{-5}$ at most. We further extrapolate in $m$ the quantities we calculate to improve their accuracy.

The DMRG is done on system of finite size, and the calculated longitudinal current cannot be directly compared with the theoretical prediction from [9]. We thus extend the fermionization approach of $[9]$ to a finite size system with periodic boundary conditions (PBCs). The boundary 
conditions on the boson fields in (2)-(3) are:

$$
\begin{aligned}
& \theta_{c}(x+L)=\theta_{c}(x)+\pi \sqrt{2} \sum_{\sigma} J_{\sigma}, \\
& \theta_{s}(x+L)=\theta_{s}(x)+\pi \sqrt{2} \sum_{\sigma} \sigma J_{\sigma} \\
& \phi_{c}(x+L)=\phi_{c}(x)+\frac{\pi}{\sqrt{2}} \sum_{\sigma} N_{\sigma}, \\
& \phi_{s}(x+L)=\phi_{s}(x)+\frac{\pi}{\sqrt{2}} \sum_{\sigma} \sigma N_{\sigma},
\end{aligned}
$$

where $J_{\sigma}, N_{\sigma}$ are integers. For the fermion fields defined as $\psi_{r}(x)=\frac{e^{i \frac{\theta_{s}(x)}{\sqrt{2}}-r \sqrt{2} \phi_{s}(x)}}{\sqrt{2 \pi a}}$, where $r= \pm$, this leads to the following boundary conditions:

$$
\psi_{r}(x+L)=\psi_{r}(x) e^{i \pi\left[1+\sum_{\sigma} \sigma\left(J_{\sigma}-r N_{\sigma}\right)\right]},
$$

where the extra factor $e^{i \pi}$ is coming from the commutation relation between the Klein factor and the number operator $[35,36]$. These boundary conditions ensure that the ground state of the pseudofermion Hamiltonian is always unique with zero total momentum. When the total particle number is fixed, that is $\sum_{\sigma} N_{\sigma}=0$ and the current $\sum_{\sigma} J_{\sigma}=0$, Eq. (11) implies that the fermions have antiperiodic boundary conditions. Their dispersion relation is thus $\epsilon_{n}=$ $\sqrt{\left[\pi(2 n+1) u_{s} K_{s} / L\right]^{2}+\Delta_{s}^{2}}$ with $n$ an integer and $\Delta_{s}$ the gap. Thus, for $\lambda>\lambda_{c}=\frac{2 \Delta_{s}}{u_{s}}$ the expression of the antisymmetric current becomes

$$
J_{s}(\lambda)=\frac{u_{s} K_{s}}{2}\left[\frac{\lambda}{2 \pi}-\frac{2}{L} \mathrm{E}\left(\frac{L \sqrt{\left(\frac{\lambda u_{s} K_{s}}{2}\right)^{2}-\Delta_{s}^{2}}}{2 \pi u_{s} K_{s}}+\frac{1}{2}\right)\right],
$$

where $\mathrm{E}(x)$ is the integer part. We note that the current starts to deviate from the linear response of the Meissner state when $\lambda>\sqrt{\lambda_{c}^{2}+(2 \pi / L)^{2}}$ and if we plot $L\left[\frac{\lambda}{2 \pi}-J_{s}(\lambda) /\left(u_{s} K_{s}\right)\right]$ versus $L \sqrt{\lambda^{2}-\lambda_{c}^{2}} /(2 \pi)$ we shall find a function taking even integer values with jumps whenever the scaling variable is an odd integer.

In the region of $\Omega$ where this bosonization approach is valid, we could use this finite size behavior to extract the critical $\lambda$ using small system sizes: i.e. up to $L=24$. This is illustrated in Fig. 1 where for $\Omega=0.125 t$ the expression (12) is compared with DMRG data by adjusting the parameter $\lambda_{c}=0.408$ to obtain the best collapse. This result is in good agreement with what we obtain by looking for example at the sharp drop in the spin current as a function of $\lambda$ at larger sizes $(L=48)$ on a grid $\lambda=\frac{2 m}{L} \pi$ with $m$ an integer number, chosen to minimize size effects. In this case the sudden drop is located between $\lambda=0.3926$ and $\lambda=0.5236$.

In Fig. 3 we show the scaled deviation of the longitudinal current for $\Omega=0.75 t$ where a deviation from formula (12) is observed due to the fact that at increasing $\Omega$ the coupling between the legs becomes stronger and the continuum approximation leading to Eq. 3 breaks down. A fine tuning of $\Omega$ shows that deviations from continuum description start to appear above $\Omega=0.25 t$.

In conclusion, using DMRG and bosonization technique, we have studied the behavior of the leg particle current of a bosonic two leg ladder under a flux. We have obtained a finite-size scaling expression for the current near the Meissner-Vortex transition using bosonization, and we have compared it with the DMRG calculation, allowing us to find both the gap and the velocity of low energy excitations in the vortex state. In future DMRG studies, we will consider other observables such as the rung current, the density correlations, and the momentum distribution and we will similarly analyze their finite size scaling.

We thank F. Ortolani for the DMRG code. M.D.D. and M.L.C. acknowledges partial supports from PRIN-2011 "Collective Quantum Phenomena: from strongly correlated system to quantum simulators". 


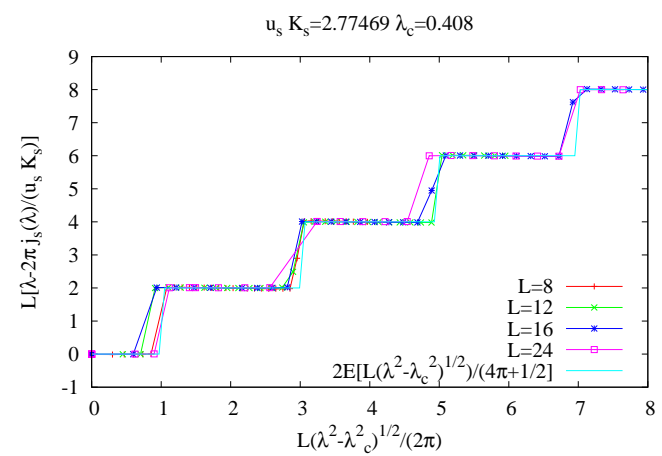

Fig. 2. Plot of the scaled deviation of the current from the linear response in the vortex phase, $L\left[\frac{\lambda}{2 \pi}-J_{s}(\lambda) /\left(u_{s} K_{s}\right)\right]$, versus the scaling variable $L \sqrt{\lambda^{2}-\lambda_{c}^{2}} /(2 \pi)$ for system sizes $L=8,12,16,24$ and $\Omega=0.125 t$. The scaled deviation has plateaus on even integer values. Jumps between plateaus occur whenever the scaling variable is an odd integer. The theoretical prediction from bosonization is plotted as the cyan line.

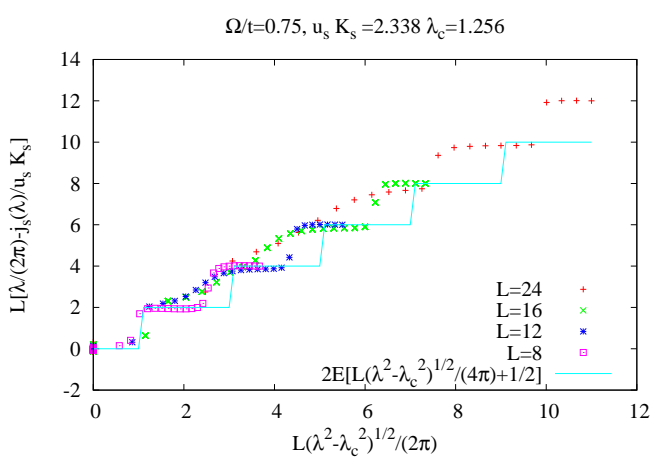

Fig. 3. Plot of the scaled deviation of the current from the linear response in the vortex phase, $L\left[\frac{\lambda}{2 \pi}-J_{s}(\lambda) /\left(u_{s} K_{s}\right)\right]$, versus the scaling variable $L \sqrt{\lambda^{2}-\lambda_{c}^{2}} /(2 \pi)$ for system sizes $L=8,12,16,24$ and $\Omega=0.75 t$. The scaled deviation doesn't show net plateaus and jumps between plateaus become less pronounced. The theoretical prediction from bosonization is plotted as the cyan line.

We acknowledge the collaboration of the IT Center, University of Pisa, for making available significant computational resources.

\section{References}

1. M. Lewenstein, A. Sanpera, V. Ahufinger, B. Damski, A. Sen De, U. Sen, Ann. Phys. (N. Y.) 56, $243(2007)$

2. K. Osterloh, M. Baig, L. Santos, P. Zoller, M. Lewenstein, Phys. Rev. Lett. 95, 010403 (2005)

3. J. Ruseckas, G. Juzeliūnas, P. Öhberg, M. Fleischhauer, Phys. Rev. Lett. 95, 010404 (2005)

4. Y. Lin, K. Jimenez-Garcia, I.B. Spielman, Nature 471, 83 (2011)

5. V. Galitski, I.B. Spielman, Nature (London) 494, 49 (2013)

6. M. Kardar, Phys. Rev. B 33, 3125 (1986)

7. E. Granato, Phys. Rev. B 42, 4797 (1990)

8. Y. Nishiyama, Eur. Phys. J. B 17, 295 (2000)

9. E. Orignac, T. Giamarchi, Phys. Rev. B 64, 144515 (2001)

10. M.C. Cha, J.G. Shin, Phys. Rev. A 83, 055602 (2011)

11. G.I. Japaridze, A.A. Nersesyan, JETP Lett. 27, 334 (1978)

12. V.L. Pokrovsky, A.L. Talapov, Phys. Rev. Lett. 42, 65 (1979) 
13. P.A. Bobbert, R. Fazio, G. Schön, G.T. Zimanyi, Phys. Rev. B 41, 4009 (1990)

14. S.E. Korshunov, Europhys. Lett. 9, 107 (1989)

15. M. Atala, M. Aidelsburger, M. Lohse, J. Barreiro, B. Paredes, I. Bloch, Nature Physics 10, 588 (2014)

16. A. Petrescu and K. Le Hur, Phys. Rev. Lett. 111, 150601 (2013)

17. A. Dhar, M. Maji, T. Mishra, R. V. Pai, S. Mukerjee, and A. Paramekanti,Phys. Rev. A 85, 041602 (2012); A. Dhar, T. Mishra, M. Maji, R. V. Pai, S. Mukerjee, and A. Paramekanti, Phys. Rev. B 87, $174501(2013)$

18. D. Hügel, B. Paredes, Phys. Rev. A 89, 023619 (2014)

19. A. Tokuno, A. Georges, New J. Phys. 16, 073005 (2014)

20. M. Piraud, Z. Cai, I.P. McCulloch, U. Schollwöck, Phys. Rev. A 89, 063618 (2014)

21. L. Barbiero, M. Abad, A. Recati, Magnetic phase transition in coherently coupled Bose gases in optical lattices, arXiv:1403.4185 (2014)

22. S. Peotta, L. Mazza, E. Vicari, M. Polini, R. Fazio, D. Rossini, J. Stat. Mech.: Theor. Exp. 2014, P09005 (2014)

23. Z. Xu, W. Cole, S. Zhang, Phys. Rev. A 89, 051604(R) (2014)

24. J. Zhao, S. Hu, J. Chang, F. Zheng, P. Zhang, X. Wang, Phys. Rev. B 90, 085117 (2014)

25. C. Hamner, Y. Zhang, M. Khamehchi, M.J. Davis, P. Engels, Spin-orbit coupled Bose-Einstein condensates in a one-dimensional optical lattice, arXiv:1405.4048 (2014)

26. M. Piraud, F. Heidrich-Meisner, I. P. McCulloch, S. Greschner, T. Vekua, U. Schollwck, arXiv:1409.7016

27. A. Petrescu, K. Le Hur, arXiv:1410.6105

28. Ahmet Keles, M. Ö. Oktel, arXiv:1411.0749

29. F.D.M. Haldane, Phys. Rev. Lett. 47, 1840 (1981)

30. M. Cazalilla, R. Citro, T. Giamarchi, E. Orignac and M.Rigol, Rev. Mod. Phys.83, 1406 (2011)

31. F. Crépin, N. Laflorencie, G. Roux, P. Simon, Phys. Rev. B 84, 054517 (2011)

32. A. Luther, Phys. Rev. B 15, 403 (1977)

33. S.R. White, Phys. Rev. B 48, 10345 (1993)

34. U. Schollwöck, Rev. Mod. Phys. 77, 259 (2005)

35. D. Senechal, An introduction to bosonization, in Theoretical Methods for Strongly Correlated Electrons, edited by D. Sénechal et al. (Springer, New York, 2003), CRM Series in Mathematical Physics

36. M.A. Cazalilla, J. Phys. B 37, S1 (2004) 